\title{
Distinction of Consciousness Fields According to Taheri from Other Conventional Physical Fields: Evaluating the Magnetic Properties of Materials
}

Mohammad Taheri

Cosmointel

Forouz Payervand

DANA R\&D

Farzad Ahmadkhanlou

University of California Irvine

Sara Torabi

University of Tehran

Farid Semsarha ( $\sim$ semsarha@alumni.ut.ac.ir)

University of Tehran https://orcid.org/0000-0001-5401-8124

Article

Keywords: Consciousness Fields, hysteresis loop, magnetic properties, physical fields

Posted Date: June 21st, 2021

DOI: https://doi.org/10.21203/rs.3.rs-618789/v1

License: (9) This work is licensed under a Creative Commons Attribution 4.0 International License.

Read Full License 
Distinction of Consciousness Fields According to Taheri from Other Conventional Physical Fields: Evaluating the Magnetic Properties of Materials

Mohammad Ali Taheri ${ }^{1}$, Firouz Payervand ${ }^{2}$, Farzad Ahmadkhanlou ${ }^{3}$, Sara Torabi ${ }^{3}$, Farid Semsarha ${ }^{3}$

1. Sciencefact R\&D Department, Cosmointel Ltd. Research Center, Ontario, Canada

2. R\&D Consultant, Tehran, Iran

3. Dept. of Mechanical and Aerospace Engineering, University of California Irvine, Irvine, CA, USA

4. Department of Plant Biology, School of Biology, College of Sciences, University of Tehran, Tehran, Iran

5. Institute of Biochemistry and Biophysics (IBB), University of Tehran, Tehran, Iran

* Correspondence: Farid Semsarha Ph.D., Institute of Biochemistry and Biophysics (IBB), University of Tehran, P.O. Box: 13145-1384, Tehran, Iran

Email: Semsarha@alumni.ut.ac.ir 


\begin{abstract}
Magnetization hysteresis loop provides important information about the magnetic properties of materials. In this study, by examining the magnetic properties of three paramagnetic and diamagnetic materials in the vicinity of the Consciousness Fields (three types 1,2 and 3), we have investigated the nature of the Consciousness Fields in comparison with magnetic field. The magnetic properties of the materials under the influence of three distinct Consciousness Fields have changed significantly. Furthermore, the Consciousness Field 1 of the present study (with originally named Faradarmani by Taheri) has changed the magnetic properties of materials toward their physically inherent state in standard laboratory conditions. Based on the conditions and the results of the present study, it can be concluded that the Consciousness Fields are inherently neither electric nor magnetite fields and have a completely different and distinct impact on materials and their properties.
\end{abstract}

Keyword: Consciousness Fields, hysteresis loop, magnetic properties, physical fields 


\section{Introduction}

Magnetic properties of materials depend on their composition and microstructure. Those properties that depend mainly on composition are intrinsic properties (Roth, 2001). Vibrating-sample magnetometer (VSM) is a versatile technique that described in 1950s for characterization of magnetic properties of materials (Foner, 1959). Based on the behavior of materials in an external magnetic field, they are usually classified into three major types: (1) diamagnetic materials such as gold are repelled when placed in a magnetic field, (2) paramagnetic materials such as platinum can be magnetized but magnetization disappears by removing the external field, and (3) ferromagnetic materials such as iron and nickel exhibit the strongest magnetic behavior and remain magnetized even after removing the external magnetic field. New magnetic phases can be induced by applying external pressure (Kamarad, 2014), temperature (Chaddah and Roy 2001) and external magnetic field (Kuwahara et al, 1995). Technical information about the magnetic properties of materials can be obtained by studying the hysteresis loop, which expresses the relationship between the magnetic field strength $(\mathrm{H})$ and magnetization of the material $(\mathrm{M})$.

The magnetic properties of pure metals and nanoparticles have long been studied in various environmental conditions. The saturation magnetization and its related magnetism alterations of very pure iron and nickel (Crangle and Goodman 1971), nonmagnetic oxides (Sundaresan et al 2006) and Porous Anodic Aluminum (PAA) (Sun et al 2013) at room temperature, and aluminum and copper at low temperature (Reekie and Hutchison 1948) have been studied. The limited observed changes are attributed to the motion of free electrons in the metal lattice and the change in the physical structure of the nanoparticle lattice as a result of the application of an external magnetic field. 
Humans have always been curious to know the world around them. There have been many attempts to explore and explain diverse physical laws. For example, Newton's law of gravity and Maxwell's electromagnetism equations. Grand unification theory (GUT) is another attempt that suggest the unification of the fundamental forces of nature and Quantum physics has shown that some physical laws extend beyond the material world (Aquino, 1999). The field concept is used frequently in physical theories. In 2000s Mohammad Ali Taheri introduced the so-called Consciousness Fields (CFs). In this theoretical concept, cosmic consciousness is the collection of consciousness or intelligence governing the universe. Consciousness is one of the three elements of the universe and is not matter nor energy (Taheri, 2013). So far, several studies have been conducted in the field of electrical activity of the human brain during communication with the Consciousness Fields (Taheri et al 2020a), using the Faradarmani Consciousness Field for treatment of animal models of Alzheimer's disease (Taheri et al 2021a) and a selected plant strain under salinity stress (Torabi et al 2020), behavior of cancer cells in culture medium and behavior of bacteria in the presence of the Faradarmani Consciousness Field (Taheri et al 2021b).

This study is the beginning of examining the third component of the universe apart from the world of matter and energy, so-called consciousness by Taheri, and examining its effect on the world of matter and energy in an experimental typical physics study in a laboratory scale with the ability to replicate and reproduce its results. Accordingly, the present study has been designed with three purposes: First, to investigate the existence of the Consciousness Fields in practice and in a purely physical study. Second, to study the difference between the theoretical nature of the Consciousness Fields and other physical fields in the experimental assay. And third, to study the type of effect of the Faradarmani Consciousness Field on the level of the target material, in comparison with other Consciousness Fields. 


\section{Materials}

The saturation magnetization of materials is one of the intrinsic properties of materials. In this study, the changes to the saturation magnetization of materials are measured in the presence of three different Consciousness Fields according to Taheri, once in the absence and once in the presence of metal shields with different thicknesses. Three categories of magnetic materials in powder form are examined: Nickel $(\mathrm{Ni})$ as ferromagnetic, Alumina (Al2O3) as quasiferromagnetic, and Copper $(\mathrm{Cu})$ as diamagnetic. The use of metal shields with different thicknesses is to obtain possible evidence of the distinction between the Consciousness Fields and other conventional physical fields. Throughout the experiments, the control samples were placed in the lower compartment with no steel shields. The test samples were placed on the upper compartment, once with no shield and once inside cylindrical stainless-steel shields. The test setup and shields specifications are presented in the following figure and table.
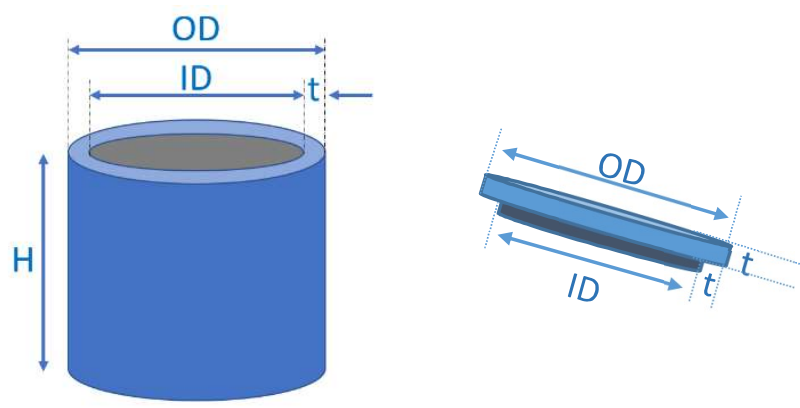

(a) 
(b)

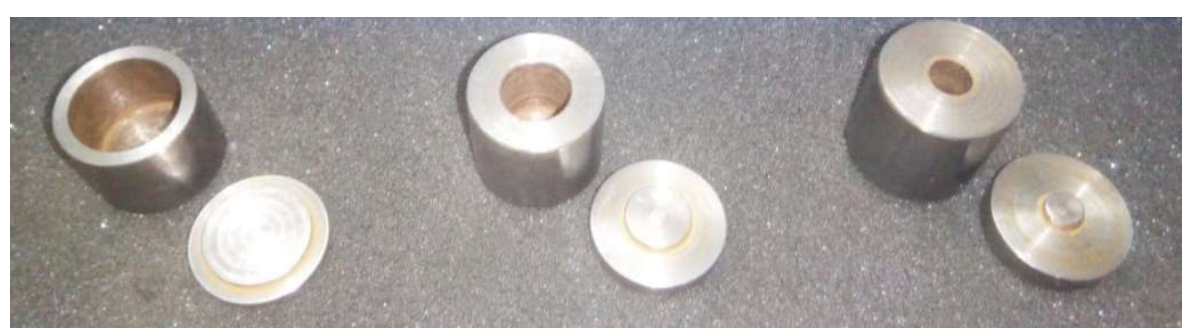

Figure 1. Schematic cylindrical metal shield dimensions (a) and three metal shields of the present study (b).

Table 1. Dimensions of the cylindrical stainless-steel shields

\begin{tabular}{cccccc}
\hline $\begin{array}{c}\text { Shield } \\
\text { Number }\end{array}$ & Material & $\begin{array}{c}\mathrm{H} \\
(\mathrm{mm})\end{array}$ & $\begin{array}{c}\text { OD } \\
(\mathrm{mm})\end{array}$ & $\begin{array}{c}\mathrm{ID} \\
(\mathrm{mm})\end{array}$ & $\begin{array}{c}\mathrm{t} \\
(\mathrm{mm})\end{array}$ \\
\hline 0 & No shield used & - & - & - & - \\
\hline 1 & Stainless-steel & 20.6 & 21.0 & 6.0 & 7.5 \\
\hline 2 & Stainless-steel & 19.0 & 21.0 & 10.0 & 5.5 \\
\hline 3 & Stainless-steel & 16.3 & 21.0 & 16.0 & 2.5 \\
\hline
\end{tabular}

\section{Method}

\subsection{Hysteresis Measurements}

The magnetic properties of the samples were measured using a Vibrating Sample Magnetometer (VSM), which operates based on Faraday's induction law. First, the sample is placed in a constant magnetic field. The applied magnetic field strength $(\mathrm{H})$ magnetizes the specimen by aligning the magnetic domains or magnetic spins of the atoms or molecules in the direction of the magnetic field. the larger the magnetic field, the sample gets more magnetized. The magnetic moment generated in the sample induces a magnetic field around the sample. At this point, when the sample vibrates up and down, the induced magnetic field changes with time based on equation 1 :

(1) $\varnothing=A H+B(4 \pi-D) M_{S} \sin \omega t$ 
Where $\mathrm{A}$ and $\mathrm{B}$ are the geometric factors associated with the set of coils, $\mathrm{D}$ and $\mathrm{M}_{\mathrm{S}}$ are the demagnetization and induction magnetization coefficients respectively, and $\omega$ is the vibration frequency of the sample.

Changes in the induced magnetic field can be observed with the induced current in a coil set. This induced current (emf) is proportional to the induced magnetization of the sample based on equation 2:

$$
\text { (2) } \text { emf }=\frac{d \emptyset}{d t}=C(4 \pi-D) M_{S} \omega \cos \omega t
$$

Where $\mathrm{C}$ is a constant.

By using VSM instrument, stronger magnetization generates larger induction current. The induced current is amplified and transmitted to a data acquisition system which is connected to a computer to record the data using related software which the results were controlled and recorded. The VSM used in this study (Figure 2) is MDKB (Iran) with the accuracy of applying the external magnetic field strength $\left(\mathrm{H}_{\mathrm{C}}\right)$ to the tenth of Orested $(\mathrm{Oe})$ and the accuracy of measuring the magnetism $(\mathrm{M})$ to the tenth of a thousand emu/g. The hysteresis curves which are the magnetization of the samples (M) versus the applied magnetic field strength (Hc) were plotted in figures 3-5.

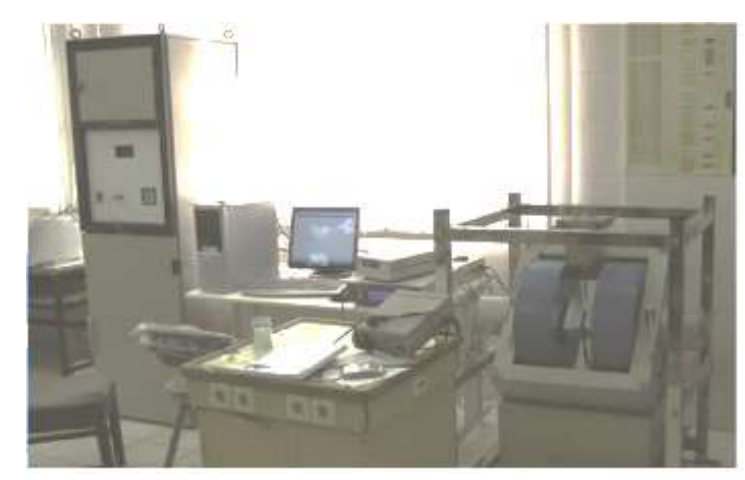

Figure 2. MDKB VSM Setup. 


\subsection{Consciousness Fields Application}

According to Taheri, a Consciousness Field initiates its function by establishing a connection between three components: (1) the Cosmic Consciousness Network (Whole Consciousness), (2) the announcer, which is the Cosmointel website and its specialized team for this study, and (3) the subject under study, which are the samples of nickel, alumina, and copper in this study. Complete information on the basics of Consciousness Field theories and applications is available on the Cosmointel research website, and some of it in accordance with the results of the present study has been used in the discussion section.

Researchers from anywhere in the world can use the complimentary service at any time to initiate the Consciousness Fields for their studies. This can be done by visiting the Cosmointel website (www.cosmointel.com) and completing the application form for intervention in the assign announcement section. Researcher should provide the details of the sample under study including its number and the exact time and place of the experiment. Also, the type of Consciousness Field should be specified. After that, intervention of the required Consciousness Field allocates in accordance with that study. 


\section{Results}

In the present study, three materials with different magnetite characteristic are used and corresponding influence of three distinct Consciousness Fields $\mathrm{CF}_{1}, \mathrm{CF}_{2}$, and $\mathrm{CF}_{3}$ have been investigated. All the sample materials are standard laboratory grades. The magnetic properties such as magnetization of the samples are presented in Table 2. Hysteresis plots of the samples are shown in Figures 3,4 and 5.

Table 2. Specification and magnetization of the samples with different Consciousness Fields treatment in this study

\begin{tabular}{|c|c|c|c|c|c|c|c|c|c|}
\hline Used CF & Material & Characteristic & Weight/mg & $\begin{array}{l}\text { Treatment } \\
\text { time/days }\end{array}$ & $\begin{array}{c}\text { Interval }^{1} \\
\text { /days }\end{array}$ & $\begin{array}{c}\text { Name } \\
\text { according to } \\
\mathrm{CF} / \text { shield } \\
\mathrm{No.}^{2}\end{array}$ & $\begin{array}{c}\mathrm{M} \\
(\mathrm{emu} / \mathrm{g})\end{array}$ & $\Delta \mathrm{M}$ & $\begin{array}{c}\text { Change } \\
\%\end{array}$ \\
\hline \multirow{4}{*}{$\mathrm{CF}_{1}$} & \multirow{4}{*}{$\mathrm{Ni}$} & \multirow{4}{*}{ Ferromagnetic } & \multirow{4}{*}{5} & \multirow{4}{*}{7} & \multirow{4}{*}{3} & $\mathrm{Ni00}$ & 54.113 & 0.000 & 0 \\
\hline & & & & & & Ni11 & 57.291 & 3.1780 & 6 \\
\hline & & & & & & Ni12 & 56.804 & 2.6910 & 5 \\
\hline & & & & & & Ni13 & 57.783 & 3.6530 & 7 \\
\hline \multirow{5}{*}{$\mathrm{CF}_{1}$} & \multirow{5}{*}{$\mathrm{Al}_{2} \mathrm{O}_{3}$} & \multirow{5}{*}{$\begin{array}{l}\text { Quasi- } \\
\text { ferromagnetic }\end{array}$} & \multirow{5}{*}{3} & \multirow{5}{*}{14} & \multirow{5}{*}{7} & Alumina00 & 0.020 & 0.00 & 0 \\
\hline & & & & & & Alumina11 & 0.0150 & -0.005 & -25 \\
\hline & & & & & & Alumina12 & 0.00760 & -0.0124 & -62 \\
\hline & & & & & & Alumina13 & 0.00550 & -0.0145 & -73 \\
\hline & & & & & & Alumina10 & 0.013 & -0.0070 & -35 \\
\hline \multirow{5}{*}{$\mathrm{CF}_{2}$} & \multirow{5}{*}{$\mathrm{Al}_{2} \mathrm{O}_{3}$} & \multirow{5}{*}{$\begin{array}{l}\text { Quasi- } \\
\text { ferromagnetic }\end{array}$} & \multirow{5}{*}{3} & \multirow{5}{*}{4} & \multirow{5}{*}{11} & Alumina00 & 0.00283 & 0.000 & 0 \\
\hline & & & & & & Alumina20 & 0.00920 & 0.00637 & 225 \\
\hline & & & & & & Alumina21 & 0.01289 & 0.01000 & 355 \\
\hline & & & & & & Alumina22 & 0.00188 & -0.00095 & -34 \\
\hline & & & & & & Alumina23 & 0.04029 & 0.03746 & 1323 \\
\hline \multirow{4}{*}{$\mathrm{CF}_{1} / \mathrm{CF}_{2} / \mathrm{CF}_{3}$} & \multirow{4}{*}{$\mathrm{Al}_{2} \mathrm{O}_{3}$} & \multirow{4}{*}{$\begin{array}{l}\text { Quasi- } \\
\text { ferromagnetic }\end{array}$} & \multirow{4}{*}{3} & \multirow{4}{*}{4} & \multirow{4}{*}{11} & Alumina00 & 0.00283 & 0.000 & 0 \\
\hline & & & & & & Alumina10 & 0.00237 & -0.00046 & -16 \\
\hline & & & & & & Alumina20 & 0.00920 & 0.00637 & 225 \\
\hline & & & & & & Alumina30 & 0.00832 & 0.00549 & 1939 \\
\hline \multirow{4}{*}{$\mathrm{CF}_{1} / \mathrm{CF}_{2} / \mathrm{CF}_{3}$} & \multirow{4}{*}{$\mathrm{Cu}$} & \multirow{4}{*}{ Diamagnetic } & \multirow{4}{*}{3} & \multirow{4}{*}{4} & \multirow{4}{*}{11} & $\mathrm{Cu} 00$ & 0.00540 & 0.000 & 0 \\
\hline & & & & & & $\mathrm{Cu} 10$ & 0.00418 & -0.00122 & -23 \\
\hline & & & & & & $\mathrm{Cu} 20$ & 0.00467 & -0.00073 & -13.5 \\
\hline & & & & & & $\mathrm{Cu} 30$ & 0.01692 & 0.01152 & 213 \\
\hline
\end{tabular}

1. Time between the end of treatment and beginning of the VSM test.

2. The first and second numbers stand for Consciousness Field numbers and shield numbers respectively. For example, Alumina21 mean Alumina sample with Consciousness Field 2 and shield number 1 . Cu00 means copper sample with no Consciousness Field applied and no shield, which is the control sample for copper. 
As shown in Figure 3, by applying the Consciousness Field $1\left(\mathrm{CF}_{1}\right)$ to the nickel samples, the magnetization value of these samples increased.

a

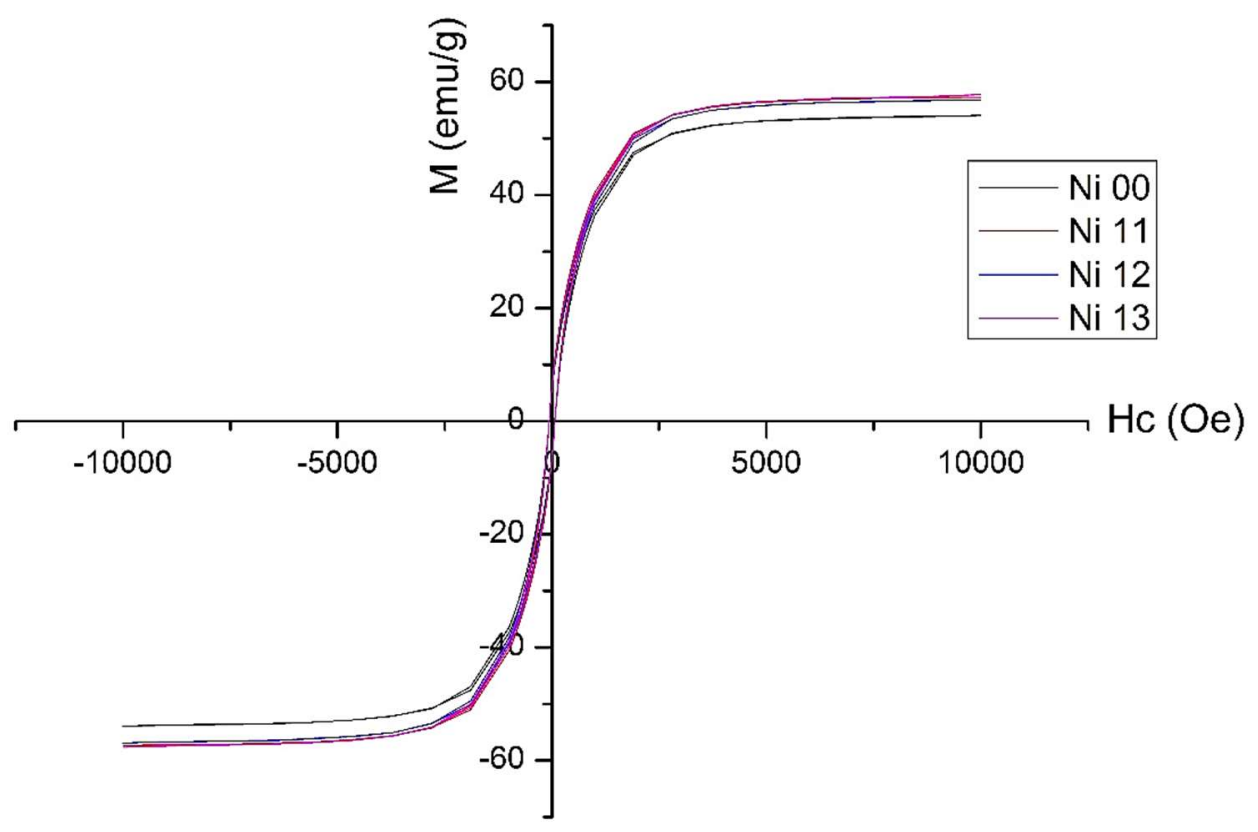

b

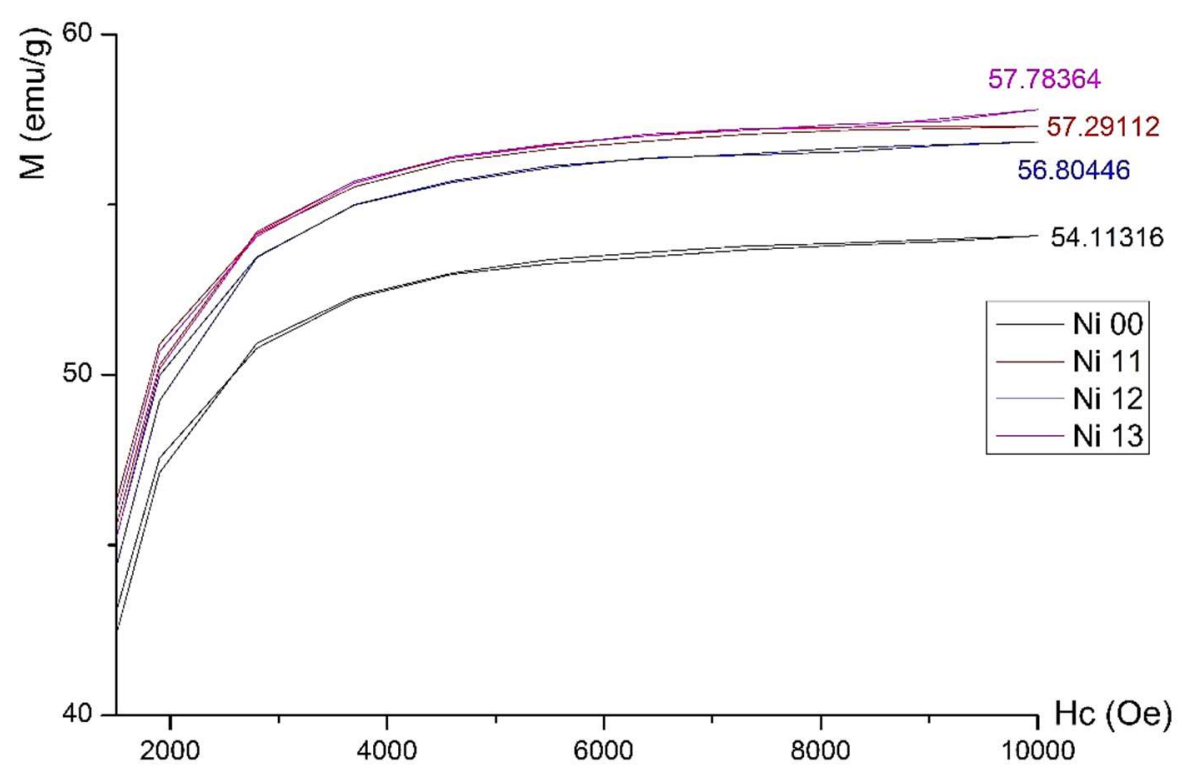

Figure 3. Hysteresis plot of the Ni samples (a) and closer view at upper part (b). 
The presence of a metal shield changes the effect of the Consciousness Field 1 on the treated samples. Except for Ni12 sample, as the thickness of the shield increases, the magnetization value of the samples decreases. Moreover, influence of Consciousness Field 1 besides two other Consciousness Fields on Alumina samples shown in Figure 4. 
a

b

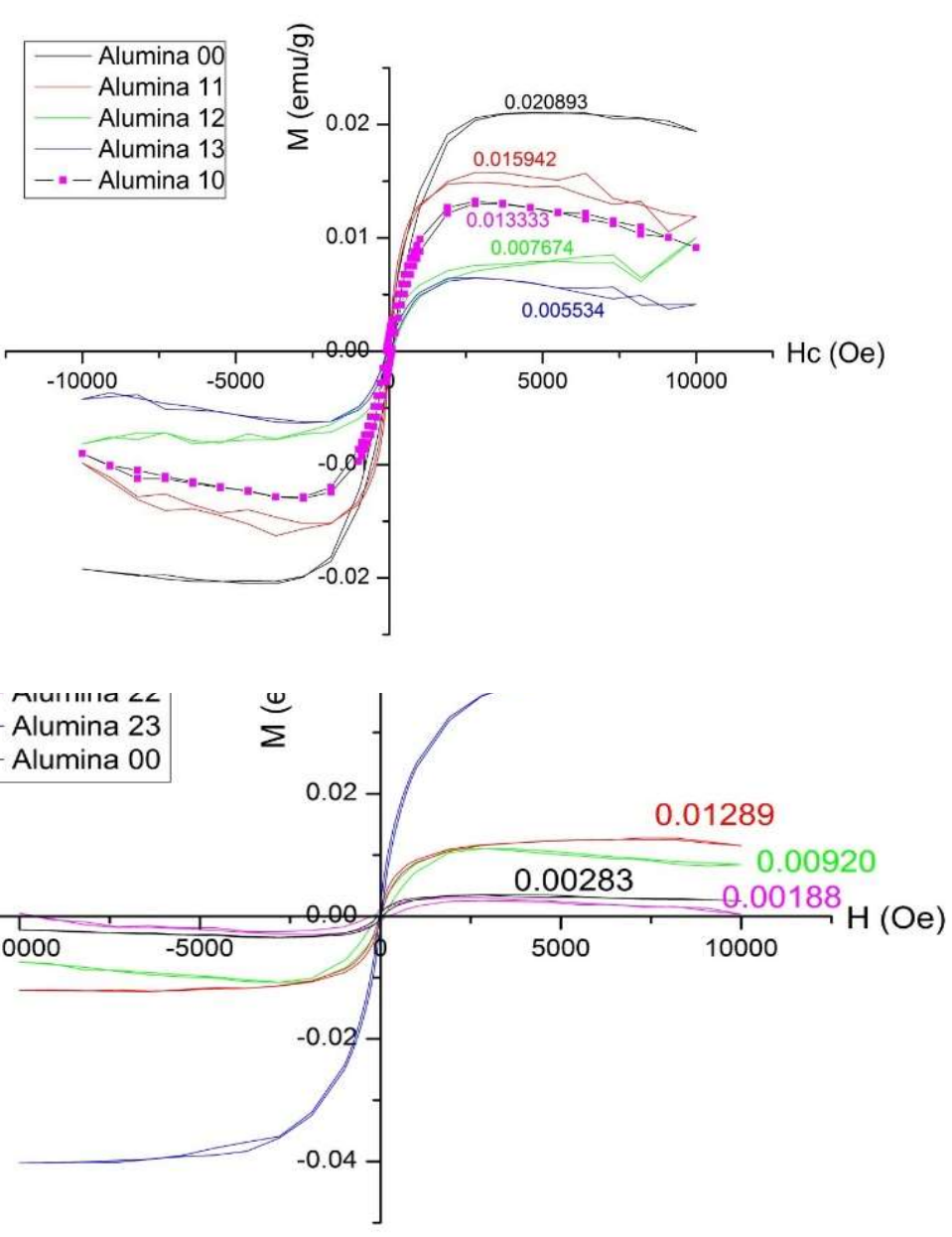

C

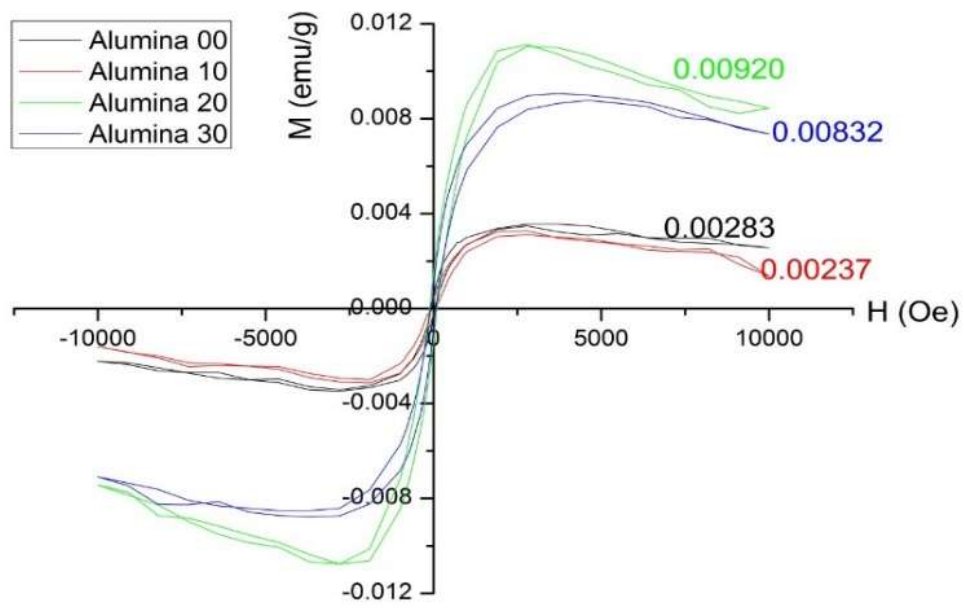

Figure 4. Hysteresis plot of the alumina samples in different shields under the influence of (a) $\mathrm{CF}_{1}$, (b) $\mathrm{CF}_{2}$, and (c) $\mathrm{CF}_{1}, \mathrm{CF}_{2}$ and $\mathrm{CF}_{3}$ in comparison with the control. 
As can be seen in Figure 4a, by applying the Consciousness Field $1\left(\mathrm{CF}_{1}\right)$ to alumina quasiferromagnetic samples, the magnetization value of these samples decreases. Similar to Ni samples test results, the presence of a metal shield changes the effect of $\mathrm{CF}_{1}$ on the alumina samples: as the thickness of the shield increases, the magnetization value of the samples decreases. On the other hand, by comparing the control (Alumina00) and the sample affected by the $\mathrm{CF}_{1}$ without shield (Alumina10) with Alumina12 and Alumina13 samples, one concludes that the shield thickness has impact on the response to $\mathrm{CF}_{1}$ and the change in overall magnetization.

Figure $4 \mathrm{~b}$ shows the change in magnetization of the alumina samples placed in different shields under the influence of Consciousness Field $2\left(\mathrm{CF}_{2}\right)$. Contrary to the previous behavior of alumina samples under the influence of the $\mathrm{CF}_{1}$, the alumina samples under the influence of $\mathrm{CF}_{2}$ show completely opposite behavior, i.e., the magnetization increases significantly with increasing the shield thickness, except for the sample inside shield 2. Similar to samples responses to $\mathrm{CF}_{1}, \mathrm{CF}_{2}$ was more effective on the change in magnetization for the alumina samples in shields 1 and 3. And finally, Figure $4 \mathrm{c}$ represents the change in magnetization of the alumina samples placed in different shields under the influence of Consciousness Field $3\left(\mathrm{CF}_{3}\right)$. Unlike $\mathrm{CF}_{1}$ and remarkably more than $\mathrm{CF}_{2}, \mathrm{CF}_{3}$ increases the magnetization of the alumina samples. 


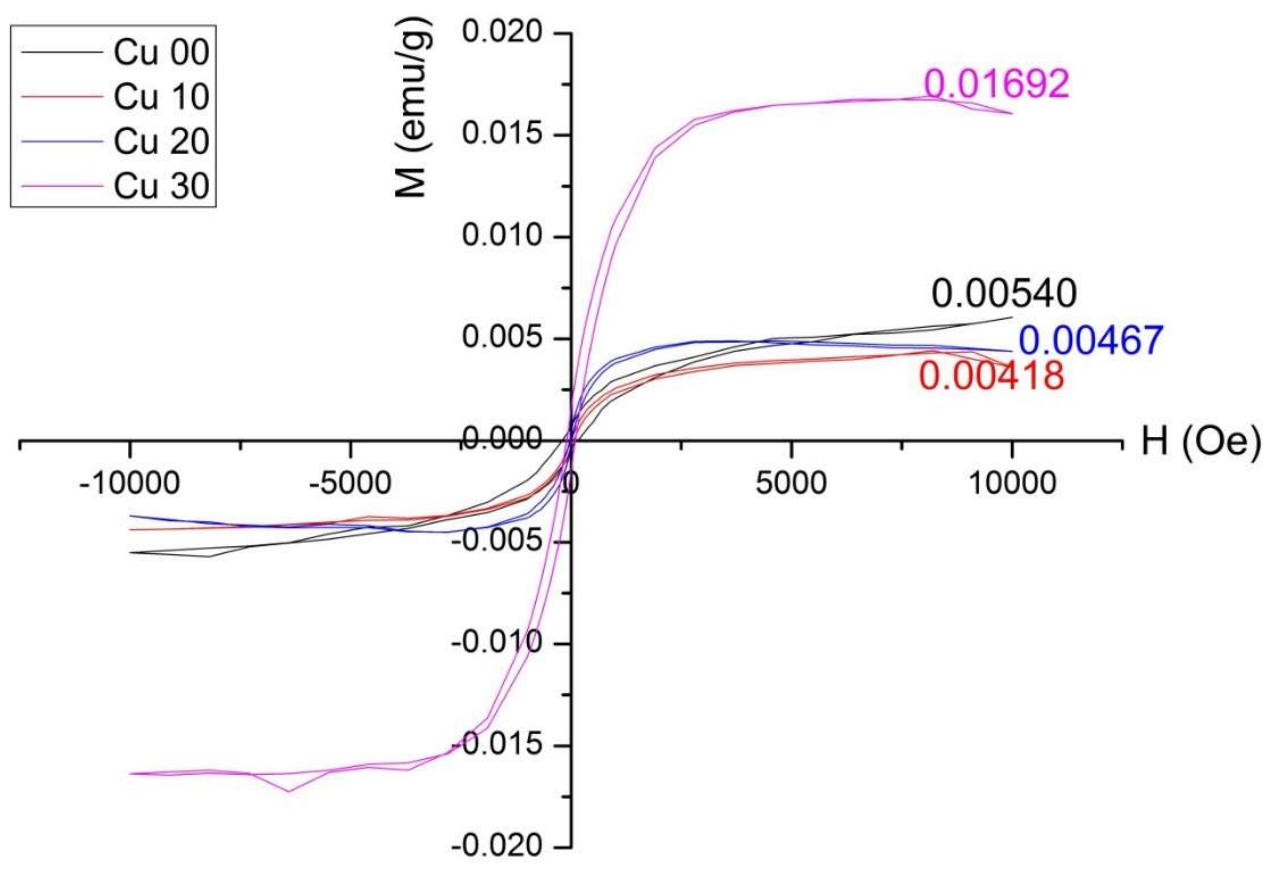

Figure 5. Hysteresis plot of the $\mathrm{Cu}$ samples.

As shown in Figure 5, the effects of $\mathrm{CF}_{1}$ and $\mathrm{CF}_{2}$ on the copper samples are similar and are accompanied by a decrease in samples magnetization. The effects of $\mathrm{CF}_{3}$ on the copper and alumina samples are similar in increasing the magnetization remarkably, in contrast to the effects of $\mathrm{CF}_{1}$ and $\mathrm{CF}_{2}$. 


\section{Discussion}

The magnetization value of the nickel control sample $(\mathrm{Ni00})$ at $30^{\circ} \mathrm{C}$ temperature is about 54.1 $\mathrm{emu} / \mathrm{g}$, which is very close to the reported value of $54.7 \mathrm{emu} / \mathrm{g}$ in the literature at this temperature. Crangle and Goodman (1971) reported the magnetization of nickel samples at $293 \mathrm{Kelvin}\left(19.9^{\circ} \mathrm{C}\right)$ is about $55.1 \mathrm{emu} / \mathrm{g}$ and it increases with decreasing the temperature, and reaches to about 58.6 emu/g at 4 Kelvin. The magnetization value of the nickel sample in shield 3 (Ni13), with the lowest thickness, reached $57.8 \mathrm{emu} / \mathrm{g}$ under the influence of the Faradarmani Consciousness Field $\left(\mathrm{CF}_{1}\right)$, which is equivalent to the observed magnetization for nickel at a temperature of about 140 Kelvin $\left(-133.1^{\circ} \mathrm{C}\right)$, based on Crangle and Goodman (1971).

In powder form, alumina exhibits ferromagnetic behavior at room temperature due to the presence of oxygen vacancies and the exchange interactions between the electron spins (Sundaresan et al 2006 and Sun et al 2013). Sintering these nanoparticles at $1400^{\circ} \mathrm{C}$ and $1 \mathrm{bar}$ pressure for one hour causes the resulting alumina mass to have a diamagnetic behavior (Sundaresan et al 2006). By applying the $\mathrm{CF}_{1}$ to alumina samples, the amount of magnetization of this quasi-ferromagnetic material was reduced. Similar to the test results with nickel samples, the presence of a metal shield changes the magnetic behavior of material under the influence of $\mathrm{CF}_{1}$, i.e., as the thickness of the shield increases the change of magnetization of samples decreases. On the other hand, the sample under influence of $\mathrm{CF}_{1}$ without shield (Alumina10) has a magnetization value between those of Alumina11 and Alumina12. This shows the presence of the steel shield itself enhances the effect of the $\mathrm{CF}_{1}$ in alumina samples to some extent. Moreover, the magnetization value obtained for Alumina13 sample at ambient temperature is $5.534 \times 10^{-3} \mathrm{emu} / \mathrm{g}$, which is comparable to the magnetization value of $3.5 \times 10^{-3} \mathrm{emu} / \mathrm{g}$ for alumina nanoparticles sintered at $500^{\circ} \mathrm{C}$ (Sundaresan et 
al 2006). This indicates that alumina under influence of $\mathrm{CF}_{1}$ is close to its intrinsic minimum magnetization value.

The Faradarmani $\mathrm{CF}\left(\mathrm{CF}_{1}\right)$ also reduces the saturation magnetism of the copper samples. $\mathrm{CF}_{1}$ improves the magnetic properties of copper as a diamagnetic material, i.e., towards its inherent state. Similar behavior was observed in the nickel and alumina samples under the influence of $\mathrm{CF}_{1}$ as well. Since the external magnetic field affects the saturated magnetism of materials and not the intrinsic magnetism (which is temperature dependent), the applied changes are only for treatment with $\mathrm{CF}_{1}$ and the reproducibility of the experiment has been investigated several times.

Based on the results of this study on the effect of $\mathrm{CF}_{2}$ on the samples, it is not possible to have definite conclusions. However, it is observed that the thickness of the shield has an effect on the rate of the change of the saturation magnetization of alumina samples under the influence of $\mathrm{CF}_{2}$. On the other hand, the effects of $\mathrm{CF}_{3}$ on all three types of materials in this study were clearly the opposite of the results observed in the presence of $\mathrm{CF}_{1}$. 


\section{Conclusions}

Hysteresis behavior of materials under the influence of a magnetic field provides significant information of intrinsic material properties. According to the theory of Consciousness Fields introduced by Taheri, the function of Faradarmani Consciousness Field (named $\mathrm{CF}_{1}$ in the present study) is to repair, modify and treat the system under study in order to achieve the optimal conditions of that system according to the general rules of the ecosystem governing it (Taheri et al 2013). The goal of this study is to investigate the distinction of CFs, according to Taheri, from other physical fields. The effects of three different types of CFs on three materials with different magnetic characteristics have been investigated. The Faradarmani Consciousness Field $\left(\mathrm{CF}_{1}\right)$ is considered as the main Consciousness Field of this study. $\mathrm{CF}_{2}$ and $\mathrm{CF}_{3}$ have been considered to better distinguish and summarize the effectiveness of $\mathrm{CF}_{1}$. By considering conditions applied in this research and the results in the presence of $\mathrm{CF}_{1}$ in accordance with the theory of the Consciousness Field function, as well as the results of the influences of $\mathrm{CF}_{2}$ and $\mathrm{CF}_{3}$, the following conclusions can be made:

(1) The existence of the Consciousness Fields and their effectiveness are clearly confirmed at laboratory scale and under controlled conditions. (2) The magnetic properties of materials enhanced remarkably under the influence of Consciousness Fields interventions. More specifically, the magnetic properties of materials move towards their inherent optimal states under the influence of Faradarmani $\mathrm{CF}\left(\mathrm{CF}_{1}\right)$. (3) Since the saturation magnetization value of a material cannot be increased or decreased by applying an electric or magnetic field, the Consciousness Fields are neither electric nor magnetic fields, i.e., the Consciousness Fields are not electromagnetic fields. (4) Since the shields thicknesses have clear reductive influence on the $\mathrm{CF}_{1}$ effects on the magnetic properties of the materials, it can be concluded the Faradarmani CF is 
applied from outside of the system under study, i.e., $\mathrm{CF}_{1}$ has an external origin other than the system under study.

Based on the results of this study, the authors recommend further investigation on the effects of Consciousness Fields according to Taheri on other physical properties of materials as well as comparing their influences with different types of energies. 


\section{References}

Crangle J. and Goodman G. M.1971.The magnetization of pure iron and nickel. Proc. R. Soc. Lond. A321477-491http://doi.org/10.1098/rspa.1971.0044

Sundaresan A., Bhargavi R., Rangarajan N., Siddesh U., and Rao C. N. R. 2006. Ferromagnetism as a universal feature of nanoparticles of the otherwise nonmagnetic oxides. Phys. Rev. B 74, 161306(R)

Hui-yuan Sun, and Shu-min Yang, Hui-min Zhang, Xue Hou, Li-hu Liu, Tian-shan Wu. 2013.

Significant room temperature ferromagnetism in PAA thin films. J. Mater. Chem. C, 1, 3569.

Roth, S. Soft Magnetic Materials: Basics. 2001. Elsevier. Pages: 8684 - 8687. ISBN: 978-0-08043152-9 https://doi.org/10.1016/B0-08-043152-6/01551-5

Foner, Simon (1959). "Versatile and Sensitive Vibrating-Sample Magnetometer". Rev. Sci. Instrum. 30 (7): 548-557. Bibcode:1959RScI...30..548F. doi:10.1063/1.1716679.

Kamarad, J. (2014). Magnetic Systems: External Pressure-Induced Phenomena.

Chaddah, P., \& Roy, S. B. (2001). Vortex matter and its phase transitions. Current science, 80(8), 1036.

Kuwahara, H., Tomioka, Y., Asamitsu, A., Moritomo, Y., \& Tokura, Y. 1995. A First-Order Phase Transition Induced by a Magnetic Field. Science, 270(5238), 961-963. Retrieved January 19, 2021, from http://www.jstor.org/stable/2888104

Fran De Aquino. 1999. "Theory of Everything". arXiv:gr-qc/9910036.

Reekie J and Hutchison T. S. 1948. The Effect of Cold Working on the Magnetic Properties of Pure Metals. Phys. Rev. 74, 610. 
Taheri MA: "Human from another outlook" Interuniversal Press; 2nd Edition (September 26, 2013). ISBN-13: 978-1939507006, ISBN-10: 19395070062013.

${ }^{a}$ Taheri, M.A.; Semsarha, F.; Modarresi-Asem, F. 2020. An Investigation on the Electrical Activity of the Brain during Faradarmani Connection in the Fara-Therapist Population. Preprints, 2020090679 (doi: 10.20944/preprints202009.0679.v1).

${ }^{a}$ Taheri, Mohammad Ali and Torabi, Sara and Nabavi, Noushin and Semsarha, Farid. 2021. Influence of Faradarmani Consciousness Field (FCF) on Spatial Memory and Passive Avoidance Behavior of Scopolamine Model of Alzheimer Disease in Male Wistar Rats. Available at SSRN: https://ssrn.com/abstract $=$

${ }^{\mathrm{b}}$ Taheri, MA, Semsarha, F, Mahdavi, M, Afsartala, Z and Amani, Laleh, 2020. The Influence of the Faradarmani Consciousness Field on the Survival and Death of MCF-7 Breast Cancer Cells: An Optimization Perspective. Available at SSRN: https://ssrn.com/abstract $=$

${ }^{\mathrm{b}}$ Taheri MA, Zarrini GH, Torabi S, Nabavi N, Semsarha F. 2021. Influence of Fara-darmani Consciousness Field on Bacterial Population Growth. bioRxiv 2021.01.08.426007; doi: https://doi.org/10.1101/2021.01.08.426007

Torabi S, Taheri MA and Semsarha F. 2020. Alleviative effects of Faradarmani Consciousness Field on Triticum aestivum L. under salinity stress [version 1; peer review: awaiting peer review]. F1000Research 2020, 9:1089 (https://doi.org/10.12688/f1000research.25247.1) 